\title{
Deviation from the formulated target weight of ingredients loaded into high milk yield cow recipes on California dairies
}

\author{
Y. Trillo, ${ }^{*} \dagger$ A. Lago, $\ddagger$ and N. Silva-del-Río* ${ }^{1}$ \\ *Veterinary Medicine Teaching and Research Center, 18830 Road 112, Tulare, CA 93274 \\ †Department of Population Health and Reproduction, School of Veterinary Medicine, University of California, Davis, One Shields Avenue, \\ Davis 95616 \\ łDairyExperts, Tulare, CA 93274
}

\begin{abstract}
Nutrient composition of the feed and formulated ration often differ depending on uncertainties in DM content and nutrient composition of ingredients, as well as from feeder errors during loading. The objective of this study was to describe the deviation from target weight for the high-producing cow ration (HCR) and premix (HCP) on 26 California dairies ranging in size from 1,100 to 6,900 cows. Records from a consecutive 12-mo period were extracted from FeedWatch 7 (Valley Agricultural Software Inc., Tulare, CA), a feeding management software. Variables extracted and studied were date, recipe type, recipe number, ingredient, loading sequence, target weight, weight, and tolerance level (TL, deviation allowed per ingredient during loading). Based on the distribution of the deviation from target weight for the 8 most common ingredients, loading accuracy (quartile 1; small: $|<10| \mathrm{kg}$; medium $|10|$ to $|20| \mathrm{kg}$; large $|>20| \mathrm{kg}$ ), loading precision (interquartile range $=$ quartile 3 to 1 ; small: $<20 \mathrm{~kg}$; medium: 20 to $40 \mathrm{~kg}$; large $>40 \mathrm{~kg}$ ), and extreme observations (quartile 3; small: $|<25| \mathrm{kg}$; medium: $|25|$ to $|40| \mathrm{kg}$; large: $|>40| \mathrm{kg}$ ) were described. Descriptive statistics were conducted with SAS 9.4 (SAS Institute Inc., Cary, NC). The median TL assigned to ingredients across dairies ranged from 0 to $90 \mathrm{~kg}$. At the ingredient level, the TL allowed a deviation from the median ingredient target weight of 0 to $2(53.9 \%),>2$ to $5(25.5 \%),>5$ to $10(11.6 \%)$, or $>10 \%(8.9 \%)$. A total of $2.5 \%$ of the loads did not reach the target weight set by the TL, ranging from 0.1 to $21.1 \%$ loads across dairies. Ingredient deviation from the formulated target weight across dairies was below target $49.1 \%$ of the time $[<-10(2.5 \%),-10$ to
\end{abstract}

Received November 10, 2015.

Accepted March 2, 2016.

${ }^{1}$ Corresponding author: nsilvadelrio@ucdavis.edu
$<-5(4.8 \%),-5$ to $<-2(8.9 \%),-2$ to $<0 \%(32.8 \%)]$ or at or above target $50.9 \%$ of the time $[0(3.9 \%),>0$ to $2(36.7 \%),>2$ to $5(8.9 \%),>5$ to $10(1.2 \%),>10 \%$ $(0.2 \%)$. Five dairies loaded ingredients with adequate accuracy (small to medium, quartile 1) and adequate precision (small to medium, interquartile range), but accuracy and precision were very poor on 3 dairies (large, quartile 1 and interquartile range). Rolled corn and almond hulls were loaded with adequate precision (small to medium, interquartile range) on a minimum of $64 \%$ of the dairies and adequate accuracy (small, quartile 1) on at least $68 \%$ of the dairies. In contrast, alfalfa hay, corn silage, and canola were loaded with poor precision (large, interquartile range) on a minimum of $60 \%$ of the dairies. There was a large variation within and across dairies on the deviation from target weight. Readjusting the TL settings might reduce the deviation from target weight. On 5 dairies, feeders were able to load ingredients with minimal deviation from target weight, setting achievable goals for the industry. Based on loading errors, opportunities exist to improve feeder performance on California dairies.

Key words: feeding management software, loading deviations from target, tolerance level

\section{INTRODUCTION}

Feed is the highest expense on a dairy. From 2011 to 2014, feed cost represented 61 to $64 \%$ of the total production cost on South Valley California dairies (CDFA, 2014). Research advances in past decades has facilitated the development of advanced mathematical models for ration formulation that accurately predict the performance of dairy cows based on the nutrient composition of their ration feed. These tools enable dairy nutrition consultants to formulate rations that cost the least while maximizing the efficiency of feed to milk conversion. However, the nutrient composition of the fed ration often differs from the formulated ration 
as a result of errors associated with weighing ingredients into a mixer box, as well as uncertainties in DM content and nutrient composition of the ingredients (Buckmaster and Muller, 1994; St-Pierre and Weiss, 2015). On 7 California commercial dairies, the observed variation between the fed and formulated recipe was important $(\mathrm{CV}>5 \%)$ on 29 to $79 \%$ of recipes studied for NDF, CP, fat, Ca, and P (Silva-del-Río and Castillo, 2012). Similarly, James and Cox (2008) reported high variability in $\mathrm{CP}$ and $\mathrm{P}$ content between the fed and formulated recipe. It has been reported that dayto-day variability in nutrient composition was not as large as the variability observed between the fed and formulated recipe (Sova et al., 2014). The observed variability in TMR nutrient composition might have implications in regard to milk yield (Rossow and Aly, 2013; Sova et al., 2014). Due to these uncertainties associated with the feeding process, nutrition consultants often times add a safety margin by formulating rations that exceed requirements for critical nutrients such as CP. The downside of this practice is the potential for a higher feeding cost as well as an increase in nutrient excretion, especially those with environmental impact such as $\mathrm{N}$.

Incorporating new technologies, such as a feed management software (FMS), may help dairy producers minimize the variation in nutrient composition (James and Cox, 2008). A 2009 California feeding management survey indicated that $44 \%$ of the dairy producers had incorporated a FMS into their operations (Silva-del-Río et al., 2010). This technology assists with recipe preparation, inventory management, and feeder performance monitoring. The mixer box has a scale indicator that displays the type and amount of ingredients that should be loaded per recipe, the final weight loaded per ingredient, and the start and end time of each loading action is transmitted through an antenna to the main computer. The time and amount of feed delivered per pen is recorded. This information can be used to generate reports based upon loading and delivery errors, mixing time, time between loads, and loading and delivery sequence of ingredients. Most FMS users reported to find value in the loading errors reports that could be used to evaluate the efficiency of feeders (James and Cox, 2008; Silva-del-Río et al., 2010). Control charts could also be used as a tool to monitor feed management on dairies (Stewart et al., 2011). However, no industry standard exists for an acceptable loading error. To the best of our knowledge, only one study reported loading errors from 7 Virginia dairies (James and Cox, 2008). Thus, the objective of our study was to describe loading deviations from target within and across 26 California dairies throughout a 12-mo period.

\section{MATERIALS AND METHODS}

\section{Data Collection and Dairies}

Twenty-six California dairy cattle farms using FeedWatch 7 [Valley Agricultural Software Inc. (VAS), Tulare, CA] as their FMS for at least 1 yr were enrolled in the study. A 12-mo data backup was obtained from the FMS for each farm. The final data set included information from Jan 2012 to May 2014. California dairy nutrition consultants and VAS personnel assisted with dairy identification. Enrolled dairies were located in the San Joaquin Valley and ranged in size (lactating and dry cows) from 1,100 to 6,900 cows. Each dairy was given a number according to its herd size, from largest (dairy 1) to smallest (dairy 26). Dairies 1 to 6 had over 4,000 cows, dairies 7 to 20 had between 2,000 and 4,000 cows, and dairies 21 to 26 had less than 2,000 cows. Records included information from 2 recipes, high cow ration (HCR; including 511,554 ingredient loads) and high cow premix (HCP; including 72,726 ingredient loads). A description of feeding variables among dairies in the study is presented in Table 1.

\section{Assembly and Structure of the Data Set}

The consultant version of FeedWatch 7 was used to extract records from the setup function and user reports. Data were transferred to an excel spreadsheet (Microsoft Office Excel 2010, Microsoft Corp.,

Table 1. Description of feeding variables for high cow ration and high cow premix based on median values per dairy during a 12 -mo period on 26 California dairies

\begin{tabular}{|c|c|c|c|c|c|c|}
\hline Item & \multicolumn{3}{|c|}{ High cow ration $(\mathrm{n}=26)$} & \multicolumn{3}{|c|}{ High cow premix $(\mathrm{n}=20)$} \\
\hline Recipe loads/day (no.) & 6 & 2 & 14 & 2 & 1 & 4 \\
\hline Ingredient loads/day (no.) & 43 & 16 & 108 & 9 & 4 & 19 \\
\hline Recipe load weight (kg) & 10,055 & 4,785 & 17,998 & 15,613 & 8,548 & 24,298 \\
\hline Feeders (no.) & 4 & 1 & 6 & 3 & 1 & 6 \\
\hline
\end{tabular}


Redmond, WA) to create a database for analysis. The variables extracted included date, recipe, recipe drop number, ingredient, loading sequence, target weight, weight, tolerance level (TL), and feeder ID. A description of some of the variables obtained from the FMS is shown below.

- Target ingredient weight: the expected weight that should be loaded.

- Ingredient weight: the weight read by the mixer box scale after loading each ingredient.

- Recipe load number: the number that identifies each recipe load.

- Ingredient type: Over 44 types of ingredients were used in HCR and HCP recipes across all dairies throughout the 12-mo study. Fifteen ingredients were deemed most common, as they were used in at least half of the dairies: premix $(\mathrm{n}=26$ dairies), alfalfa hay $(\mathrm{n}=26)$, corn silage $(\mathrm{n}=26)$, rolled corn $(\mathrm{n}=25)$, almond hulls $(\mathrm{n}=25)$, liquids (molasses, water, and whey; $\mathrm{n}=24$ ), whole cottonseed $(\mathrm{n}=23)$, mineral-vitamins $(\mathrm{n}=21)$, canola $(\mathrm{n}=20)$, dry distillers grains $(\mathbf{D D G} ; \mathrm{n}=$ 16), wet distillers grains (WDG; $\mathrm{n}=15$ ), straw $(\mathrm{n}=14)$, corn gluten feed $(\mathrm{n}=14)$, wheat silage $(\mathrm{n}=14)$, and by-pass fat $(\mathrm{n}=14)$. These ingredients represented $77 \%$ of the total ingredient loads. Results presented by ingredient type only include information from the 15 most common ingredient types used in HCR and HCP recipes.

- TL settings: To avoid overloading ingredients, the FMS assigns a TL to each commodity. After reaching the TL, if there is a pause of $5 \mathrm{~s}$ or longer the FMS will register the new weight as the next ingredient of the recipe.

- Feeder identification: the unique ID given to each employee operating the FMS.

\section{Calculations}

Deviation from the median recipe load target weight allowed by the TL was calculated for each dairy as (a) kilograms, TL assigned to ingredients within a recipe load; and (b) percentage, ( $\Sigma$ ingredient TL within a recipe load $/ \Sigma$ ingredient target formulated weight with the same recipe load number) $\times 100$.

Deviation from the median ingredient type target weight allowed by the TL was calculated for each dairy and ingredient type as (a) kilograms, TL assigned to each ingredient type across dairies; and (b) percentage, (TL per ingredient type/median formulated target weight by ingredient type) $\times 100$.

Deviation from TL for ingredient loads not reaching the target weight set by the TL was calculated as (a) kilograms, [(formulated target weight $-\mathrm{TL})-($ weight loaded)]; and (b) percentage: [(formulated target weight - TL) - (weight loaded)/(formulated target weight $\mathrm{TL})] \times 100$.

Deviation from recipe load target weight was calculated as the absolute value and real value for each dairy as (a) kilograms, (weight loaded per recipe load - target weight per recipe load); and (b) percentage, (weight loaded per recipe load - target weight per recipe load)/ target weight per recipe load $\times 100$.

The final deviation from target weight was calculated for the 15 most common ingredient types as (a) kilograms, (weight loaded per ingredient type - target weight per ingredient type); and (b) percentage, [(weight loaded per ingredient type - target weight per ingredient)/target weight per ingredient type] $\times 100$.

The proportion of loads with a deviation from target greater than $2 \%$ for each day of the week was evaluated. Dairies with a coefficient of variation $>10 \%$ were considered to have a dissimilar percentage by day of the week and were reported.

For each dairy, the cost of ingredients included in HCR and HCP was obtained from the FMS records. Three dairies $(1,4$, and 5$)$ had no records for ingredient cost; consequently, only information from $17 \mathrm{HCP}$ and 23 HCR were used to evaluate recipe load cost deviations. The cost per metric tonne of the target recipe was calculated as ( $\Sigma$ ingredient target weight $\times$ ingredient cost)/total target weight per recipe load. The cost per metric tonne of the recipe loaded was calculated as $(\Sigma$ ingredient weight $\times$ ingredient cost)/total weight per recipe load.

Based on the distribution of the deviation from target weight across dairies for the 8 most common ingredients (alfalfa hay, almonds hulls, canola, corn silage, liquids, premix, rolled corn, and whole cottonseed) loading accuracy [based on quartile $1\left(\mathbf{Q}_{1} ; 25\right.$ th percentile)], loading precision [based on interquartile range (IQR) $=$ quartiles 3-1], and extreme observations [based on quartile $3\left(\mathbf{Q}_{3} ; 75\right.$ th percentile)] were described. Each of these variables was classified based on their quartile distribution among dairies as small, medium, or large deviation from target rounded to the nearest figure in 5 -unit increments.

- $\mathrm{Q}_{1}$ was classified as small $\left(\mathrm{Q}_{1}=|<10| \mathrm{kg} ; 52.0 \%\right)$, medium $\left(\mathrm{Q}_{1}=|10|\right.$ to $\left.|20| \mathrm{kg} ; 38.3 \%\right)$, or large $\left(\mathrm{Q}_{1}\right.$ $=|>20| \mathrm{kg} ; 9.7 \%)$.

- $\mathrm{Q}_{3}$ was classified as small $\left(\mathrm{Q}_{3}=|<25| \mathrm{kg} ; 42.4 \%\right)$, medium $\left(\mathrm{Q}_{3}=|25|\right.$ to $\left.|40| \mathrm{kg} ; 34.7 \%\right)$, or large $\left(\mathrm{Q}_{3}\right.$ $=|>40| \mathrm{kg} ; 16.3 \%)$.

- IQR $\left(\mathrm{Q}_{3}-\mathrm{Q}_{1}\right)$ was classified as small (IQR $<20 \mathrm{~kg}$; $49.0 \%$ ), medium (IQR $=20$ to $40 \mathrm{~kg} ; 34.7 \%$ ), or large (IQR >40 kg; 16.3\%). 


\section{Data Interpretation}

To interpret study findings, additional information on feeding management practices was obtained for some dairies through interviews with dairy nutritionists, VAS personnel, or by direct interaction with feeders on dairies.

\section{Data Analysis}

Descriptive statistics were calculated with the PROC MEANS and PROC UNIVARIATE procedures of SAS 9.4 (SAS Institute Inc., Cary, NC). Percentiles were computed using the PCTLDEF $=4$ option in the output statement of the PROC UNIVARIATE. The relationship between deviation from target in kilograms and percentage by dairy was evaluated using the PROC CORR procedure of SAS 9.4.

\section{RESULTS AND DISCUSSION}

\section{Data Screening}

There were no feeding records for 2 consecutive months and for 40 nonconsecutive days on dairy 2 and 11 , respectively. This could be explained by equipment breakdown, communication problems between the software and mixer box, or unintentional deletion of computer records. On dairy 6, recipe loads prepared with the stationary mixer box (20,498 ingredient loads, $62 \%$ of dairy 6 observations) had no recipe load number information. Therefore, all observations were used to evaluate ingredient loading deviations from target, but loads prepared with the stationary mixer were not evaluated at the recipe load level.

Five dairies $(2,6,18,20$, and 23) did not prepare the HCP recipe on farm. Dairy 11 had only HCP recipe records for 90 nonconsecutive days, so it was removed from the final HCP recipe analysis. Dairy 26 (herd size 1,100) did not prepare HCP recipe during the first 5 mo of the study period, so HCP recipe records from 7 mo were included in the final data set.

On dairy 14, one ingredient load reached a 10-figure number. This observation was eliminated. The FMS automatically generates a 10-figure number when apparent total scale weights are exceeded. This could be due to cell weight errors or to the front-end-loader striking excessive weight on the mixer box.

There were ingredients not loaded into the HCR or HCP recipe. Those ingredients registered a load weight of $0 \mathrm{~kg}$ (1,299 total observations). This could be explained if ingredients were listed in the recipe but were not available at the dairy. In this scenario, the feeder must advance manually or by clicker to the next ingredient. However, the movement of the feed inside the mixer box often causes the scale reading to bounce during mixing. If the magnitude of the scale bouncing is higher than the minimum scale detection, an ingredient weight record would exist even if no ingredient was loaded. In the present study, we also considered that ingredients were not weighed down when the amount loaded was $<60 \mathrm{~kg}$, the target weight was $>100 \mathrm{~kg}$, and the amount loaded represented $<10 \%$ of the expected target weight. Based on these criteria, a total of 675 ingredients were not loaded and over half of those ingredients $(53.6 \%)$ were from dairy 15.

The initial data set included information from 584,280 ingredient loads. After data screening, the final data set included a total of 488,359 ingredient loads for HCR $[$ range $=5,900$ (dairy 1) to 84,125 (dairy 2)] and 72,422 for $\mathrm{HCP}$ [range $=4,190$ (dairy 1$)$ to 6,900 (dairy 2$)$ ].

\section{TL Settings}

All dairies used the TL settings function of the FMS (Figure 1). During the 12-mo study period, the assigned TL was kept constant for all ingredients across dairies. The minimum TL assigned to an ingredient was 0 ( $\mathrm{n}$ $=15)$ or 2.3 to $9.0 \mathrm{~kg}(\mathrm{n}=11)$ and the maximum TL level ranged from 14 to $36(\mathrm{n}=14), 45(\mathrm{n}=6), 90(\mathrm{n}$ $=2)$, or $135 \mathrm{~kg}(\mathrm{n}=4)$.

The major purpose of assigning commodities with a TL is to minimize the risk of overloading expensive ingredients. During software installation, information systems technicians educated clients on the TL settings

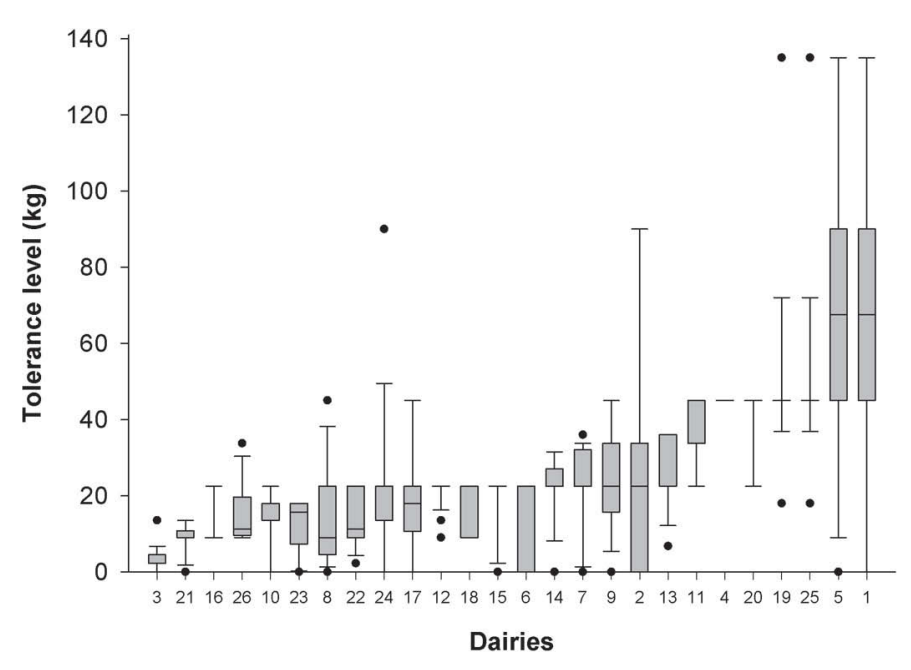

Figure 1. Boxplot of the tolerance level $(\mathrm{kg})$ assigned to the various ingredients of the high cow ration and high cow premix recipe on 26 California dairies. Data are presented sorted by 75 th percentile, and then by 50th percentile. Each boxplot shows the 50th percentile (median, line within the box), 25th and 75th percentile (box), 10th and 90th percentiles (whiskers), and outliers (dots). 
of the FMS. It is at software installation time when most users decide the TL of ingredients [personal communication with C. Lew, VAS, Tulare, CA]. Settings of $0 \mathrm{~kg}$ for TL could be explained due to dairy producers considering irrelevant to assign TL to some ingredients, or the fact they did not know how to use the TL settings of the FMS for new ingredients. Ingredients with $0 \mathrm{~kg}$ of TL were feed additives $(\mathrm{n}=12)$, forages $(\mathrm{n}=$ $10)$, by-products $(\mathrm{n}=8)$, seasonal by-products $(\mathrm{n}=4)$, premixes $(n=3)$, or grains $(n=1$; Figure 2$)$. Most of these ingredients $(78.9 \%)$ were included in the recipe for less than 6 mo. The most common TL assigned to an ingredient was $23 \mathrm{~kg}$, used 11.1 to $91.7 \%$ on 18 of the dairies. However, it is unclear the criteria by which dairy producers assigned TL to various ingredient types. Most dairies selected TL values under $36 \mathrm{~kg}$, but some dairies were more liberal with their TL settings. Six dairies assigned a similar TL to all ingredients (IQR $=0 \mathrm{~kg}$ ), and 4 dairies chose various TL (IQR $=23$ to $45 \mathrm{~kg}$ ). One dairy assigned the same TL, $45 \mathrm{~kg}$, to all ingredients.

\section{Deviation from Target Weight Allowed by TL}

The TL added to $<200(\mathrm{n}=14), 200$ to $400(\mathrm{n}=8)$, or $>400 \mathrm{~kg}(\mathrm{n}=4)$ for HCR represented 0.4 to $2.3,1.9$ to 6.9 , and 3.3 to $4.6 \%$ of deviation from the median target weight, respectively. Similarly, the TL added to $<200(\mathrm{n}=15), 200$ to $400(\mathrm{n}=4)$, or $>400 \mathrm{~kg}(\mathrm{n}=1)$ for $\mathrm{HCP}$ represented 0.2 to $1.2,1.5$ to 4.2 , and $2.8 \%$ of deviation from the median target weight, respectively. The TL could potentially introduce at least a $4 \%$ deviation from target weight for HCR on 3 dairies [dairy 5 (720 kg of TL), 19 (405 kg of TL), and $25(315 \mathrm{~kg}$ of TL)] and on 1 dairy for HCP [dairy 25 (360 kg of TL)].

At the ingredient level, the TL allowed a deviation of $0(8.7 \%),>0$ to $2(45.2 \%),>2$ to $5(25.5 \%),>5$ to $10(11.6 \%)$, or $>10 \%(8.9 \%)$ from the median ingredient target weight. Thus, the TL needs to be carefully considered, as it had the potential to introduce a deviation from target of $>5 \%$ in more than $20 \%$ of the ingredients. In most cases, the median formulated target weight for these ingredients was under $1,000 \mathrm{~kg}$. However, some ingredients with a median formulated target weight of over $1,000 \mathrm{~kg}$ had $>5 \%$ of deviation allowed by the TL [liquids $(3 / 6)$, rolled corn $(2 / 4)$, wheat silage $(1 / 2)$, WDG $(1 / 3)$, DDG $(1 / 4)$, mineral-vitamins $(1 / 6)$, and alfalfa hay $(2 / 13)]$.

Five dairies had 1 ingredient (liquid, straw, or bypass fat) with an assigned TL that allowed $>30 \%$ of deviation from the median target weight. On 4 of these dairies, the deviation was explained by the low median target weight set by the recipe $(23-83 \mathrm{~kg})$ rather than by the TL assigned to those ingredients $(18-23 \mathrm{~kg})$. However, on 1 dairy the TL assigned to liquids was 90 $\mathrm{kg}$ whereas the median target weight was $300 \mathrm{~kg}$. For ingredients added in small quantities, the most desirable loading method would be to weigh them before loading; thus, assigning a TL would be irrelevant for those ingredients.
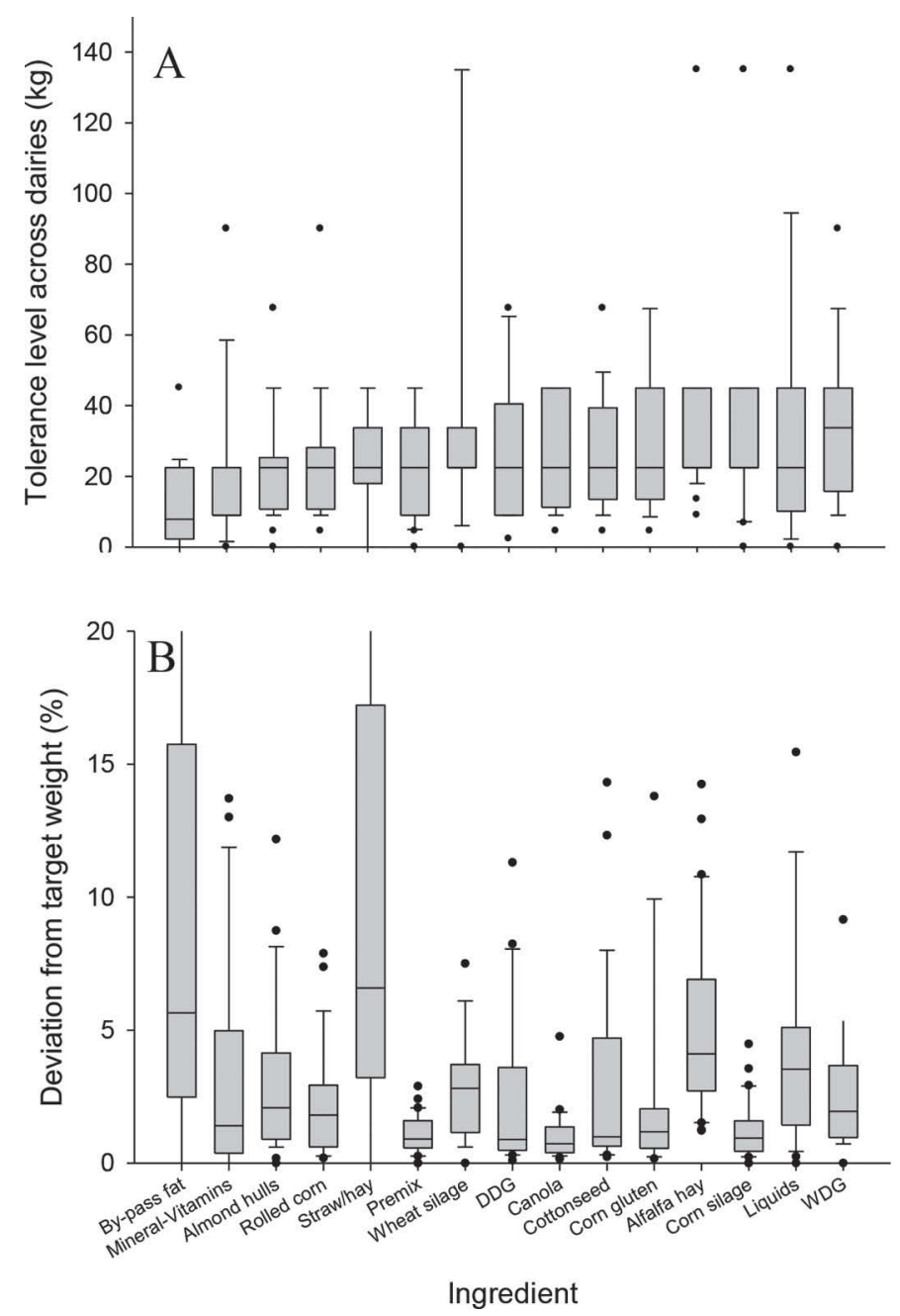

Figure 2. Boxplot of the tolerance level settings $(\mathrm{A} ; \mathrm{kg})$ and of the median deviation allowed by the tolerance level (B; \%) for ingredients included in the high cow ration and high cow premix recipes on 26 California dairies. Data are presented sorted by 75 th percentile, and then by 50th percentile (A). The boxplot shows the 50th percentile (median, line within the box), 25th and 75th percentile (box), 10th and 90th percentiles (whiskers), and outliers (dots). Panel B whisker reaches $61.2 \%$ for by-pass fat and $36.1 \%$ for straw/hay. The deviation allowed by the tolerance level was calculated per ingredient and dairy as follows: $[\Sigma$ recipe ingredient tolerance level $(\mathrm{kg}) / \Sigma$ recipe ingredient target $(\mathrm{kg})] \times 100 . \mathrm{DDG}=$ dried distillers grains; $\mathrm{WDG}=$ wet distillers grains. 


\section{Loads Not Reaching the Target Weight Set by TL}

A total of 12,439 times (2.5\% of the total observations) ingredients were loaded under the target weight set by TL. This represented 0.1 to $21.1 \%$ loads of feed per dairy (Figure 3 ). The number of loads not reaching the TL by up to $50 \mathrm{~kg}[5-80(\mathrm{n}=11$ dairies $), 128-500$ $(\mathrm{n}=10)$, or $1,230-1,830$ loads $(\mathrm{n}=5)]$ or by more than $50 \mathrm{~kg}[2-36(\mathrm{n}=12), 54-149(\mathrm{n}=10)$, and 207-319 loads $(\mathrm{n}=4)]$ ranged widely across the study dairies.

Four dairies had over 1,000 ingredient loads not reaching the TL by up to $25 \mathrm{~kg}$. The ingredients in most these cases were wheat silage (dairy 3 ); corn silage, whole cottonseed, and rice grain (dairy 12); corn silage, canola, yeast, rolled corn, and oat silage (dairy 21); and, alfalfa hay, almond hulls, corn silage, premix, and rolled corn (dairy 26). On these dairies, feeders and owners potentially could have agreed it was an acceptable practice to manually advance to the next ingredient if less than $25 \mathrm{~kg}$ were left to reach the TL. However, this practice increased the deviation from the formulated target weight by 0.2 to 0.9 percentage units. Feeders and dairy owners should be informed about the implications of routinely not reaching the TL. Based on information from the FMS, we cannot determine if this practice saved the feeder an extra trip to the commodity barn or if the feeder simply did not want to pursue the task of reaching the target weight.

On dairy 1 , some ingredient loads did not reach the TL up to $25 \mathrm{~kg}(\mathrm{n}=651)$ or from $>25$ to $50 \mathrm{~kg}$ ( $\mathrm{n}=$ 751 ). This increased the deviation from the median formulated target weight by 0.9 and 2.5 percentage units, respectively. Sorghum represented $80 \%$ of the loads not reaching the TL. This was likely explained because sorghum had $0 \mathrm{~kg}$ of TL, whereas the mean TL for all the other ingredients on this dairy was $67.5 \mathrm{~kg}$.

On 7 dairies, a total of 50 to 272 loads were below the TL by over $200 \mathrm{~kg}$. The ingredients that were most commonly underloaded were citrus by-products, liquids, and corn silage. For these ingredients, the deviation from the formulated target increased by 17.7 to 85.4 percentage units.

Over the study period, all dairies but dairy 20 had ingredients that were not loaded either 1 to $15(\mathrm{n}=$ 11), 23 to 74 ( $\mathrm{n}=12$ ), 434 (dairy 14; mostly seasonal by-products and by-pass fat), or 641 times (dairy 2; mostly liquids). We are unsure why ingredients were not loaded, but it is likely that occasionally some commodities were used up before a new truck load was delivered or one ingredient was removed from the recipe without updating the FMS. It is extremely important that dairy nutritionists and dairy managers maintain open lines of communication with feeders to understand why some ingredients are not being loaded. If adjustments need to

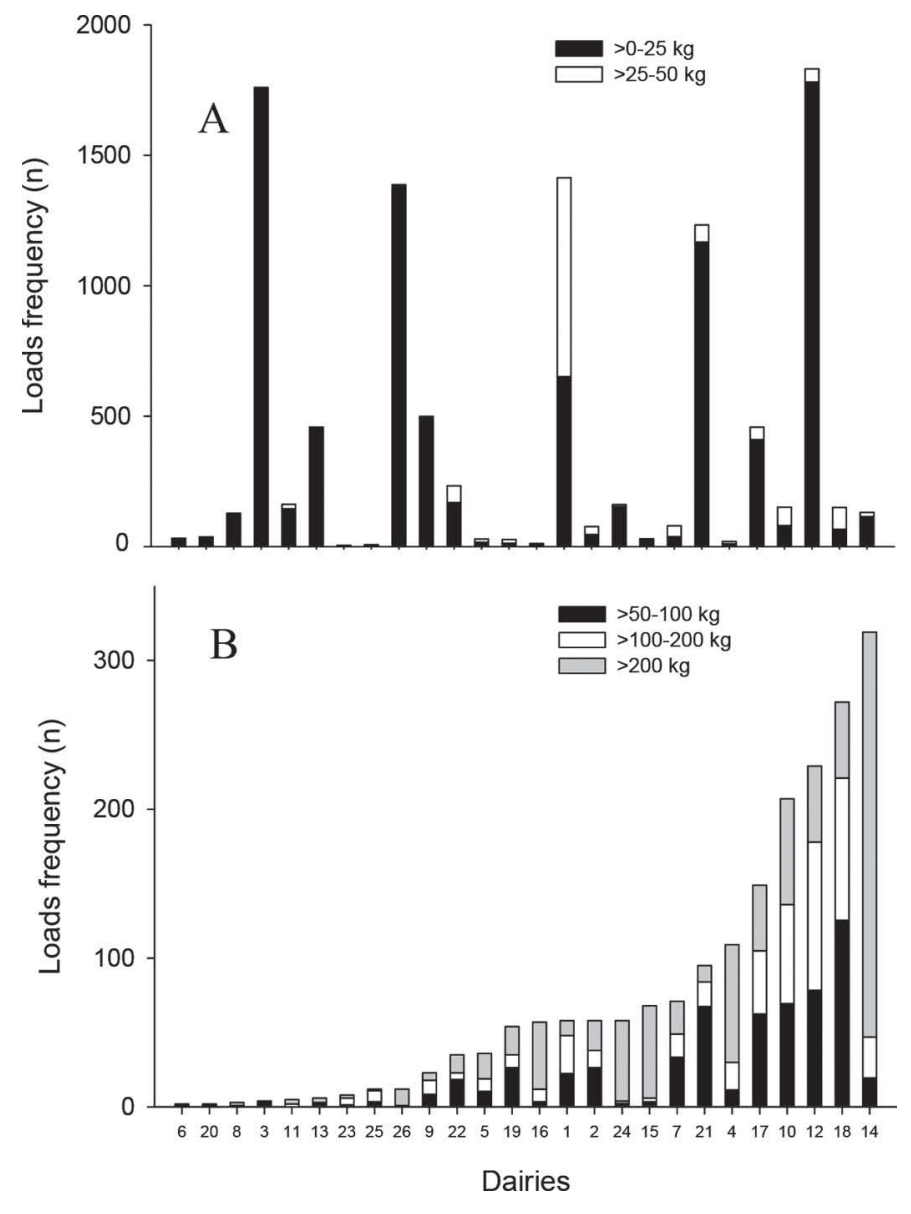

Figure 3. Frequency of loads that did not reach the target weight set by the tolerance level [by 0 to $50 \mathrm{~kg}$ (A) and by $>50 \mathrm{~kg}$ (B)] for ingredients loaded into the high cow ration and the high cow premix recipes on 26 California dairies. Data are presented sorted by the frequency of loads in B.

be made to the FMS recipe, it would be recommended to introduce those as soon as possible so feeding records can be accurately evaluated.

\section{Deviation from Target Weight by Dairy}

The deviation from target weight, as kilograms and percentage, is represented in Figure 4. Across all ingredients loaded, the deviation from the formulated target weight was $49.1 \%$ of the time below target $[<-10 \%$ $(2.5 \%),-10$ to $<-5 \%(4.8 \%),-5$ to $<-2 \%(8.9 \%)$, -2 to $<0 \%(32.8 \%)]$ and $50.9 \%$ of the time at or above target $[0 \%(3.9 \%),>0$ to $2 \%(36.7 \%),>2$ to $5 \%(8.9 \%)$, $>5$ to $10 \%(1.2 \%),>10 \%(0.2 \%)]$.

Deviation from target can be expressed in kilograms or as a percentage. When expressed in kilograms, at least $20 \%$ of the time ingredients were loaded with a deviation from target $>40 \mathrm{~kg}$ on 7 dairies $(4,7,9,10$, 14,15 , and 23 ) or $<-40 \mathrm{~kg}$ on 2 dairies ( 5 and 25 ). 

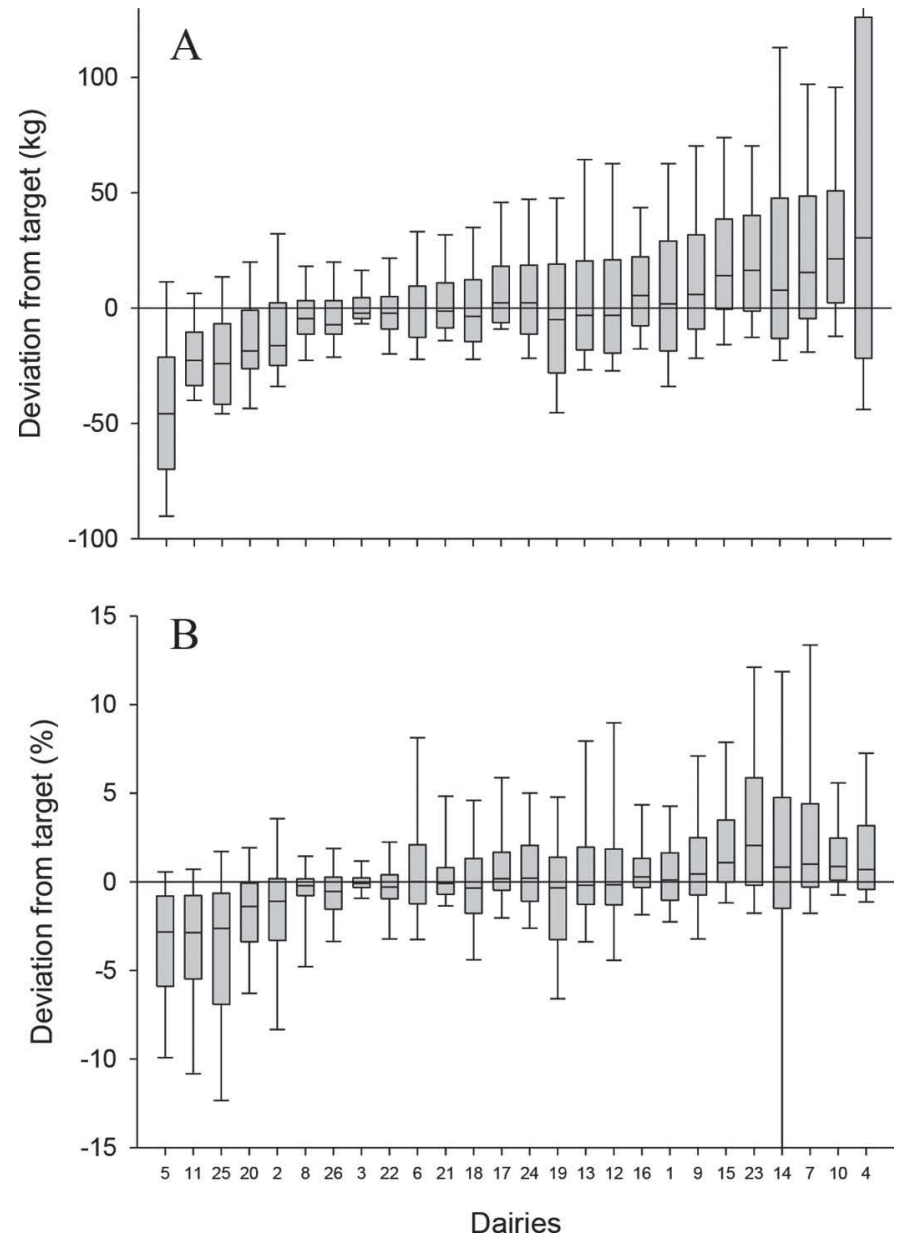

Figure 4. Boxplot distribution of the deviation from the target weight $(\mathrm{A}, \mathrm{kg} ; \mathrm{B}, \%)$ for ingredients loaded into the high cow ration and high cow premix recipes on 26 California dairies. Data are presented sorted by 75 th and then by the 50th percentile (A). The boxplot shows the 50th percentile (median, line within the box), 25th and 75th percentile (box), and 10th and 90th percentiles (whiskers). Whisker reaches: (A) $265 \mathrm{~kg}$ (dairy 4); (B) $-31 \%$ (dairy 14).

However, as a percentage, at least $20 \%$ of the time ingredient deviations from the target was $>4 \%$ on 5 dairies $(4,7,14,15$, and 23$)$ or $<-4 \%$ on 6 dairies $(2$, $5,11,19,20$, and 25). Although a significant association was noted between deviation from target weight per ingredient load, expressed as kilograms and as a percentage, the correlation coefficient was poor and only on 6 dairies was it $>0.5$. When small loads were prepared, deviation from target weight expressed as a percentage will be larger compared with big loads. This could explain why dairies 9 and 10 (the 9th and 10th largest dairies), despite having a large deviation from target in kilograms, did not show the same extend of deviation as a percentage. Likewise, 6 dairies showed an important deviation below the target weight as a percentage, but only 2 dairies when deviation was expressed as kilograms. Dairy 4 showed the largest deviation above target weight in kilograms, but dairy 23 (the 4th smallest dairy) had the largest deviation as a percentage. It is quite common that owners and nutritionists set feeder performance goals based on deviation from target as percentage rather than kilograms. Deviation from target weight expressed in percentage is a good tool to assess the extent of loading errors and their potential implications on the final nutrient composition of the recipe. However, deviation from target weight in kilograms is a better tool to monitor feeder performance. If feeder loading errors are mostly under the target weight, the assigned TL should be re-evaluated. Also, it is important to ensure that inaccuracies at loading are not due to equipment failure. The mixer box scale should be calibrated frequently and scale bouncing during mixing should be kept to a minimum. Based on our field experiences, we have observed mixer scales bouncing up to $40 \mathrm{~kg}$. This situation makes it extremely difficult for the feeder to weigh ingredients accurately. On a 2010 feeding management survey, it was reported that dairy producers and managers neglected to check the mixer box scale enough (Silva-del-Río et al., 2010).

\section{Deviation from Target Weight by Ingredient Type}

The deviation from target weight, in kilograms, for the 8 most common ingredient types is represented as a box plot in Figure 5. Straw, wheat silage, by-pass fat, mineral-vitamins, and canola were loaded in 10.0 to $14.3 \%$ of the dairies with a median deviation of $>2 \%$ from the target weight; however, by-pass fat, straw, alfalfa hay, liquids, DDG, whole cottonseed, almond hulls, corn gluten feed, and mineral-vitamins were loaded in 13.2 to $42.8 \%$ of the dairies, with a median deviation of $<-2 \%$ from the target weight. The most extreme deviation over the target weight was observed for by-pass fat on dairy 7 (21.9\%), with a median target weight of $76 \mathrm{~kg}$. The most extreme deviations under the target weight were observed for by-pass fat $[-24.3 \%$ (dairy 17 ); $-44.7 \%$ (dairy 11); $-78.7 \%$ (dairy 14 )] and mineral-vitamins $[-62.5 \%$ (dairy 17 )]. This could be explained by the low median target weight loaded for by-pass fat [247 kg (dairy 17); $23 \mathrm{~kg}$ (dairy 11); $40 \mathrm{~kg}$ (dairy 14)] and mineral-vitamins [135 kg (dairy 17)]. It is possible that most ingredients with extreme deviation from target weight were loaded as whole bags or were weighted before being added into the mixer box. In situations where the mixer was running during loading, the large deviation from target weight could be simply explained by mixer scale errors, such as a scale bouncing, rather than a lack of feeder accuracy when loading minimal quantities. 

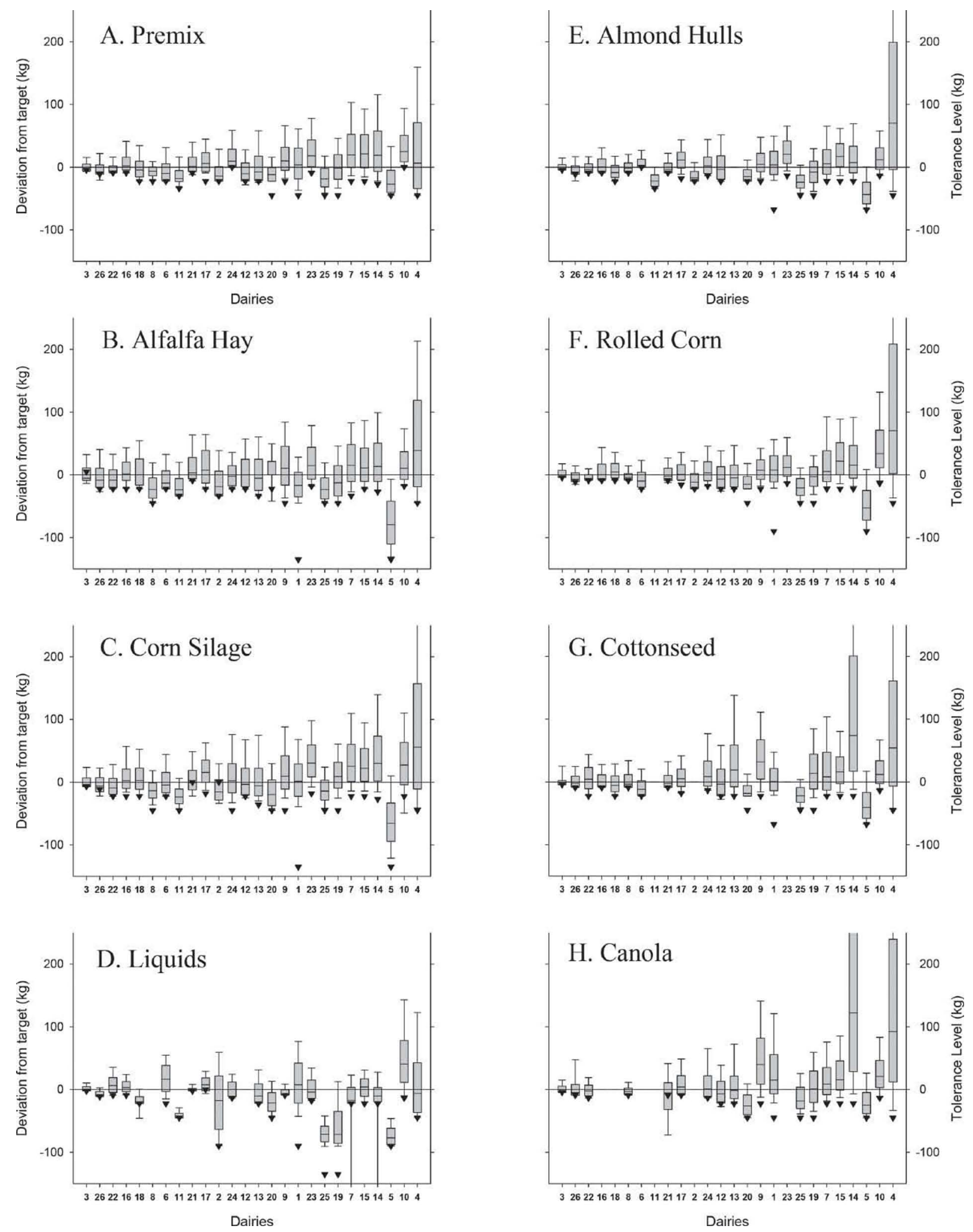

Figure 5. Boxplot distribution of the deviation from target weight for 8 of the most common ingredients of the high cow ration and high cow premix recipes during a 12 -mo period on 26 California dairies. The tolerance level is represented in the secondary y-axis as $\mathbf{\nabla}$. Data are presented sorted by overall interquartile range $\left(\mathrm{Q}_{3}\right.$ to $\left.\mathrm{Q}_{1}\right)$. The boxplot shows the 50 th percentile (median, line within the box), 25th and 75 th percentile (box), and 10th and 90th percentiles (whiskers). Whisker reaches: (C) $300 \mathrm{~kg}$ (dairy 4); (D) -640 $\mathrm{kg}$ (dairy 7 ), -440 kg (dairy 14); (E) $450 \mathrm{~kg}$ (dairy 4); (F) $380 \mathrm{~kg}$ (dairy 4); (G) $400 \mathrm{~kg}$ (dairy 14), $280 \mathrm{~kg}$ (dairy 4); (H) $500 \mathrm{~kg}$ (dairy 14; $\mathrm{Q}_{3}: 300 \mathrm{~kg}$ ), $350 \mathrm{~kg}$ (dairy 4). 


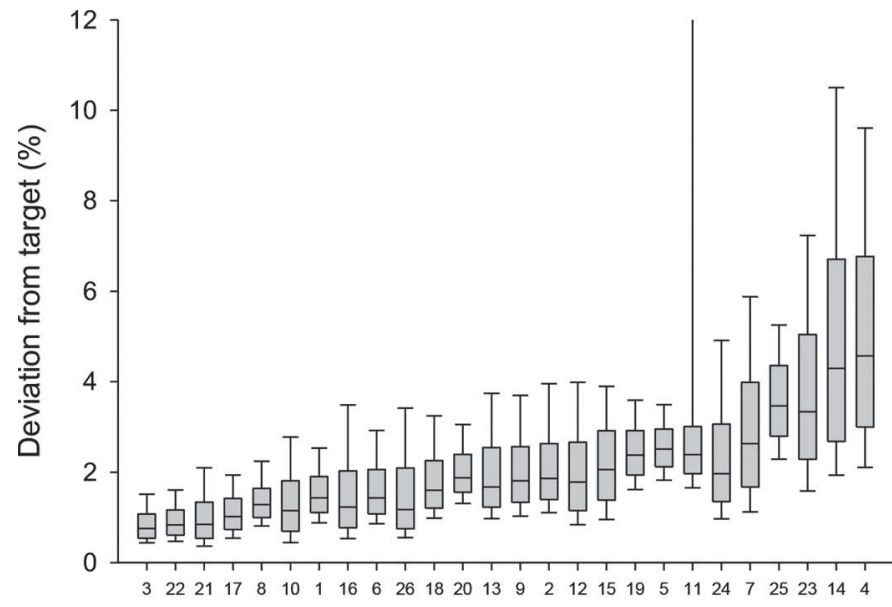

Dairies

Figure 6. Boxplot distribution of the deviation from target weight (as an absolute value, \%) for high cow ration on 26 California dairies. Data are presented sorted by 75th and then by the 50th percentile. The boxplot shows the 50th percentile (median, line within the box), 25th and 75th percentile (box), and 10th and 90th percentiles (whiskers). Upper whisker of dairy 11 reaches $33 \%$.

\section{Deviation from Target Weight for HCR and HCP Recipe}

The box plot of the absolute deviation from target for HCR as a percentage is represented in Figure 6 . The absolute deviation from target was more than $2 \%$ at least $50 \%$ of the time on 7 dairies. The real values of the median deviation for HCR recipe were either below the target weight on 10 dairies $[<-2 \%(\mathrm{n}=2),-2$ to $<-1 \%(\mathrm{n}=2),-1$ to $0 \%(\mathrm{n}=6)]$ or above the target weight on 16 dairies [>0 to $1 \%(\mathrm{n}=11),>1$ to $2 \%$ (n $=3),>2 \%(\mathrm{n}=2)]$.

The box plot of the absolute deviation from target for $\mathrm{HCP}$ as a percentage is represented in Figure 7. The absolute deviation from target was more than $2 \%$ at least $50 \%$ of the time on 3 dairies. The real values of the median deviation from target weight for HCR recipe were either below the target weight on 4 dairies $[<-2 \%(\mathrm{n}=0),-2$ to $<-1 \%(\mathrm{n}=2$; dairy 5 and 25$)$, -1 to $0 \%(\mathrm{n}=2)]$ or above the target weight on 17 dairies $[>0$ to $1 \%(\mathrm{n}=13),>1$ to $2 \%(\mathrm{n}=2),>2 \%(\mathrm{n}$ $=2)$.

Our results indicated that, on most dairies, HCP was prepared within a reasonable absolute deviation from its target as percentage. However, opportunities exist to improve the absolute deviation from target for HCR. Although HCP is designed to mix ingredients that otherwise will be added in small quantities into the HCR, 17 dairies were adding at least 1 ingredient under 225 $\mathrm{kg}$ into the HCR, most commonly straw, by-pass fat, or yeast. Only 6 dairies included at least one ingredi- ent under $225 \mathrm{~kg}$ into the HCP. Thus, dairy producers and nutritionist should evaluate if ingredients added into the HCR should rather be included into the HCP. Furthermore, it should be taken into consideration that ingredients added in small quantities often times come in bags. Feeders prefer to load whole bags as the first ingredient to avoid getting in and out of the loader during recipe preparation. This practice can compromise mixing uniformity. It is likely that feeders would be more compliant with the ingredient order at loading if they had to do it twice (median HCP recipe loads/d) versus 6 (median HCR recipe loads/d; Table 1) times per day.

\section{Deviation from Target Weight by Day of the Week}

The percentage of ingredients loaded into the HCR and HCP with a deviation from target greater than $2 \%$ by day of the week was similar $(\mathrm{CV}<10 \%)$ in 12 dairies. However, other dairies showed an important deviation (CV ranging from 10.8 to $54.8 \%, \mathrm{n}=14$ ), that in most cases $(\mathrm{n}=12)$ was explained by an extreme observation on a single day of the week.

On 5 dairies, there was an increase in the deviation from target weight on Wednesday [dairy 24 (extreme day value vs. 6 -d average): 27.7 vs $12.3 \%$ )], Thursday (dairy 25: 9.7 vs. 5.4\%), and Sunday (dairy 1: 22.5 vs. $16.4 \%$; dairy 18 : 20.8 vs. $15.7 \%$; dairy 23 : 52.8 vs. $36.8 \%$ ). Nevertheless, on 7 dairies a reduction of deviation from target weight was observed on Monday (dairy 10: 28.7 vs. 37.6\%), Wednesday (dairy 9: 27.8

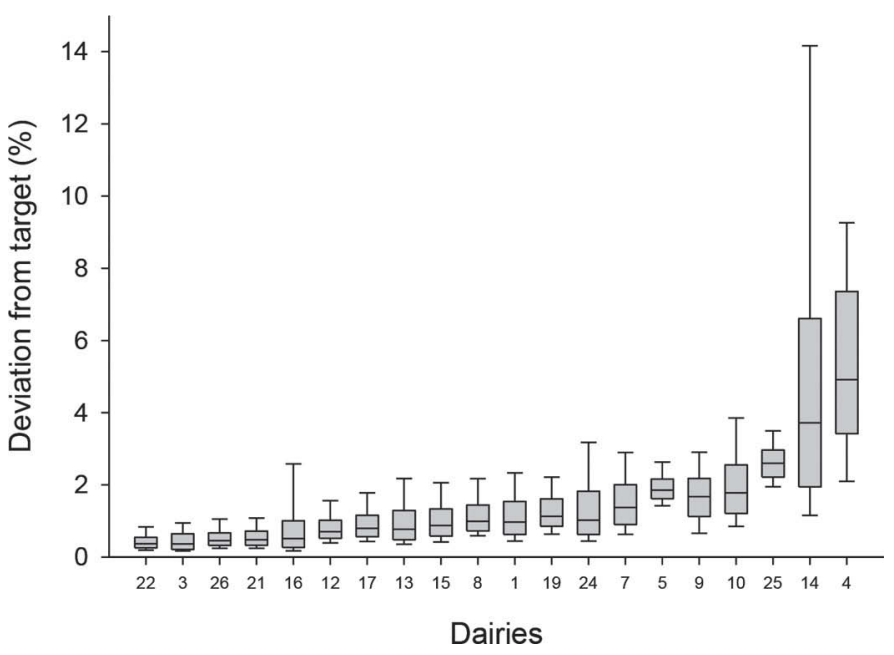

Figure 7. Boxplot distribution of the deviation from target weight (as an absolute value, \%) for high cow premix on 26 California dairies. Data are presented sorted by 75 th and then by the 50 th percentile. The boxplot shows the 50th percentile (median, line within the box), 25th and 75th percentile (box), and 10th and 90th percentiles (whiskers). 
vs. $36.0 \%$; dairy 17 : 17.6 vs. $23 \%$ ), Saturday (dairy 8: 9.0 vs. $24.9 \%$; dairy $13: 22.8$ vs. $36.1 \%$; dairy $26: 9.3$ vs. $11.9 \%$ ), and Sunday (dairy 16: 21.2 vs. $29.7 \%$ ).

Variation in deviation from target in relation to the day of the week could be related to differences in loading accuracy between the primary and secondary feeder.

In our study, we did not use the FMS information on feeder identification, as we observed that the primary user logged at least $85 \%$ of the days on 6 dairies and between 82 to $85 \%$ of the days on 8 dairies. Taking into account that most feeders get at a minimum $1 \mathrm{~d}$ off a week and at least 2 wk of vacation, those besides the primary feeder were likely logged in under the same feeder identification. On Virginia dairies, there was no significant difference in deviation from target between the primary $(1.57 \%)$ and secondary $(1.26 \%)$ feeder (James and Cox, 2008). Contrary to their initial hypothesis, secondary feeders had a numerically inferior deviation from target. James and Cox (2008) speculated that bad working habits acquired by the main feeder might have played a role in feeding errors. Information on feeders' performance may be used to establish goals and rewards among operators within a dairy; however, based on our field experience, dairy nutritionists and dairy managers pay little attention to FMS records to evaluate feeders. Thus, dairy managers are giving minimal attention to ensure feeders are logged in with their unique identification each time.

\section{Deviation from Target Recipe Cost}

The deviation from target cost for $\mathrm{HCR}$ and $\mathrm{HCP}$ recipe is represented in Figure 8. As a result of deviations from the target weight, the HCR recipe cost increased by at least $\$ 3$ per metric tonne $<5 \%$ ( $\mathrm{n}=$ $15), 5$ to $20 \%(\mathrm{n}=6)$, or $>20 \%$ (dairies 7 and 14$)$ of the times. It also decreased by $\$ 3$ per metric tonne $<5 \%(\mathrm{n}=18), 5$ to $20 \%(\mathrm{n}=4)$, or $>20 \%$ (dairy 14 ) of the times. Some dairies were consistent in the final recipe cost relative to the target cost $(\mathrm{IQR}=\$ 0.3 /$ metric tonne, dairy 3), but other dairies fluctuated largely $(\mathrm{IQR}=\$ 4.6 /$ metric tonne, dairy 14$)$.

The HCP recipe cost increased by at least $\$ 3$ per metric tonne $<5 \%$ ( $\mathrm{n}=13$ ) or 5 to $20 \%$ ( $\mathrm{n}=4$; dairies $10,14,15$, and 17) of the times or decreased by $\$ 3$ per metric tonne $<5 \%(\mathrm{n}=14), 5$ to $20 \%(\mathrm{n}=2$; dairies 14 and 15 ), and $>20 \%$ (dairy 17) of the time. The withindairy variation, based on IQR, ranged between $\$ 0.3$ (dairy 22 ) to $\$ 5.3 /$ metric tonne (dairy 17 ).

It is accepted that by overloading ingredients the recipe cost will increase. On dairy 14 , HCR recipes were mostly prepared under the target cost by at least $\$ 1$ per metric tonne, even though the feeder frequently overloaded ingredients (Figure 4). It is likely the feeder was paying attention to detail when loading costly ingredients, but not when loading relatively inexpensive ingredients. Conversely, on dairy 25, HCR and $\mathrm{HCP}$ recipes were prepared generally over the target cost per metric tonne, but very few ingredients on this dairy were loaded over the target weight as the TL was very restrictive (Figure 1). Expensive ingredients may have been loaded closer to the target than inexpensive ingredients.

Our results reflect the changes in recipe cost per metric tonne associated with deviations from the target weight. To estimate the true economic implications of loading actions, the effect on production associated with changes in nutrient composition as well as the final amount of feed per pen should have been taken into consideration. On most dairies nutritionists formulate least cost rations, thus any modification to the
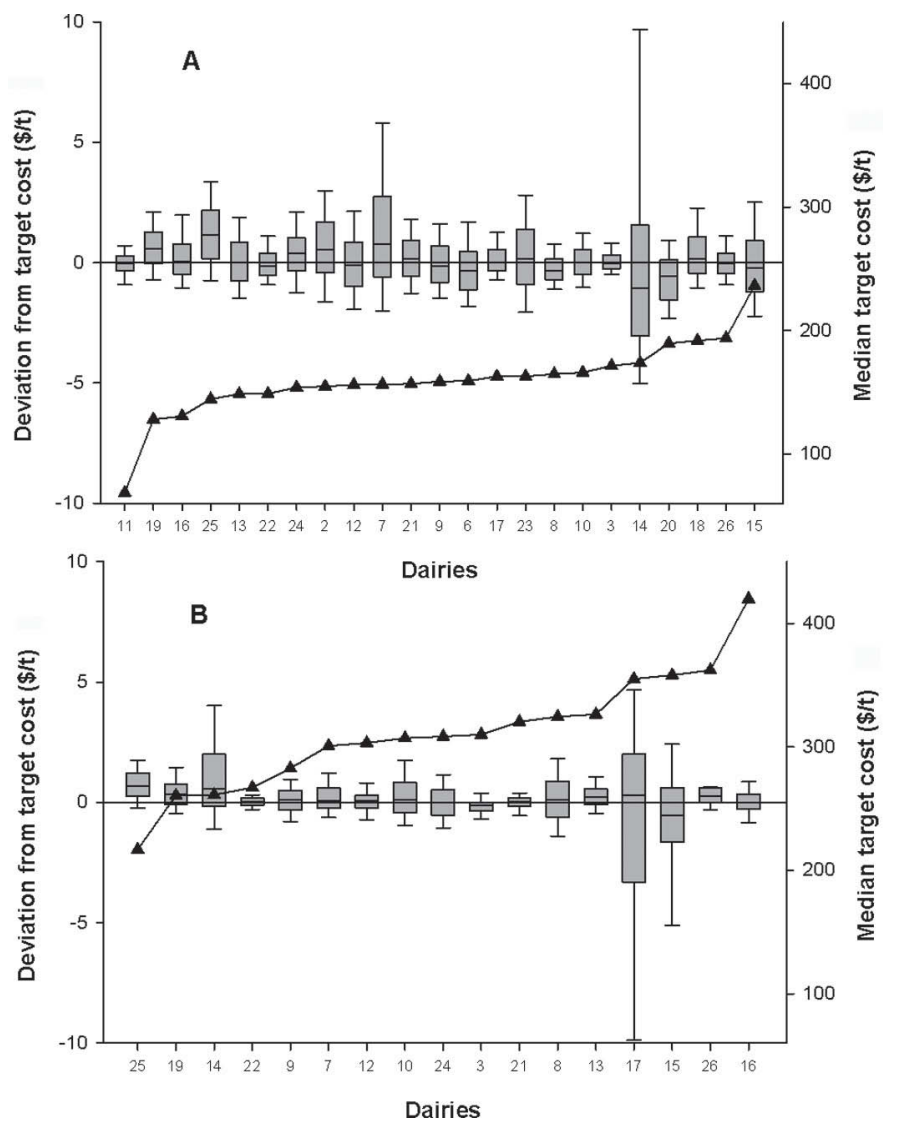

Figure 8. Boxplot distribution of the deviation from target cost by high cow ration $(\mathrm{A}, \mathrm{n}=23)$ and high cow premix $(\mathrm{B}, \mathrm{n}=17)$ recipes on California dairies. Median target cost is represented in the secondary y-axis as $\boldsymbol{\Lambda}$. Data are presented sorted by the smallest to the largest median target cost. The boxplot shows the 50th percentile (median, line within the box), 25th and 75th percentile (box), and 10th and 90th percentiles (whiskers). 
formulated recipe will most likely have a detrimental effect on income over feed cost.

\section{Distribution of the Deviations from the Target Weight Based on $Q_{1}, Q_{3}$, and IQR}

By Dairy. The deviation from target weight across dairies for the 8 most common ingredients based on $\mathrm{Q}_{1}$, $\mathrm{Q}_{3}$, and IQR is represented in Figure 9. These results provide guidelines for producers and the allied industry on achievable goals at loading. There were 5 dairies $(3$, $16,18,22$, and 26) that loaded almost all ingredients with adequate accuracy $\left(\right.$ small $\mathrm{Q}_{1}$ ) and precision (small to moderate IQR); however, on 4 dairies feeders showed inadequate accuracy (moderate to large $\mathrm{Q}_{1}$ ) by either overloading (dairy 4 and 14) or underloading ingredients (dairies 5 and 11). Loading precision was poor on dairies 4,5 , and 14 (large IQR), but good on dairy 11 (small IQR). It is likely that by reducing or eliminating TL on dairy 11 the feeder could have been accurate. Six dairies (4, 5, 7, 10, 14 and 15) loaded at least 50\% of the ingredients with a large deviation from target $\left(\mathrm{Q}_{3}:|>40| \mathrm{kg}\right)$, that represented up to a $14.5 \%$ of deviation. Dairy nutritionists and managers should actively engage with the feeder to improve loading performance on dairies with poor precision and accuracy.

The 2 dairies with the more liberal TL (0 to $135 \mathrm{~kg}$, dairies 1 and 5) were owned by the same dairyman and shared the same manager and dairy nutritionist. However, on dairy 5, deviation from target based on $\mathrm{Q}_{1}$ was large (|18| to $|44| \mathrm{kg}$ ), with most ingredients loaded under the target weight, whereas dairy 1 was relatively accurate $\left(\mathrm{Q}_{1}:|8|\right.$ to $\left.|14| \mathrm{kg}\right)$. On these 2 dairies feeders interpreted differently what the loading target was, either the one set by the TL (dairy 5) or the true target (dairy 1).

On dairy 3 , the feeder showed remarkable skills with quality precision (IQR $=0$ to $6 \mathrm{~kg})$, and accuracy $\left(\mathrm{Q}_{1}\right.$ : $|1|$ to $|7| \mathrm{kg})$ with minor deviations from target $\left(\mathrm{Q}_{3}:|4|\right.$ to $|13| \mathrm{kg})$. Conversely, the feeder on dairy 4 lacked desirable loading skills. On this dairy, precision was poor (IQR $=34$ to $208 \mathrm{~kg}$ ), accuracy was moderate to poor $\left(\mathrm{Q}_{1}:|18|\right.$ to $\left.|32| \mathrm{kg}\right)$, and extreme deviations from target were noted $\left(\mathrm{Q}_{3}:|52|\right.$ to $\left.|240| \mathrm{kg}\right)$. On dairy 3, the feeder was directly supervised by a feed manager that tracked inventory and frequently supervised feeder errors. It was likely that this close supervision influenced feeder performance. Moreover, on this dairy, minerals and feed additives were automatically added into the recipe with a micronutrient liquid dispenser, minimizing loading errors. The good accuracy and precision observed for alfalfa hay could be explained by hay processing before loading; however, it is unknown if the dairy was actually doing this. One frequent concern with increas- ing loading accuracy is the potential detrimental effect on feeder efficiency. After recipe preparation times were evaluated, loading time for dairy 3 was found to be within average (unpublished data). Thus, neglecting accuracy and precision in favor of time efficiency might be a misconception.

By Ingredient. Rolled corn and almond hulls were easy to load. On at least 64 to $80 \%$ of the dairies these ingredients were loaded precisely (IQR: $<20 \mathrm{~kg}$ ) and accurately $\left(\mathrm{Q}_{1}|<10| \mathrm{kg}\right)$. However, a total of $56.0 \%$ of the dairies loaded almond hulls with a deviation from target that ranged from 2.6 to $14.5 \%$ based $\mathrm{Q}_{3}$. Of those dairies, median inclusion rate of almond hulls ranged between 207 to $5,117 \mathrm{~kg}$, representing 2.4 to $29.6 \%$ of the as-fed weight of the recipe.

Overall, 60.0 to $61.5 \%$ of the dairies had poor precision (IQR: $>20 \mathrm{~kg}$ ) when loading alfalfa hay, corn silage, and canola. Alfalfa hay, corn silage, and canola were loaded with a large deviation from target $\left(\mathrm{Q}_{3}\right.$ : $|>40| \mathrm{kg}$ ) on $34.6,38.5$, and $45.0 \%$ of the dairies, respectively. This represented a deviation from target weight of 2.1 to $12.9 \%$ (alfalfa hay), 2.2 to $5.5 \%$ (corn silage), and 2.3 to $7.3 \%$ (canola). As expected, alfalfa hay was one of the most challenging ingredients to load accurately and precisely. Alfalfa hay particles are prone to attach to one another, forming flakes that fall together during loading. Alfalfa hay represented $5.4\left(\mathrm{Q}_{1}\right)$ to $9.9 \%\left(\mathrm{Q}_{3}\right)$ of the as-fed $\mathrm{HCR}$ recipe. Likewise, canola is an ingredient that flows rapidly from the bucket of the loader, requiring excellent skill to load accurately. Canola represented $12.5\left(\mathrm{Q}_{1}\right)$ to $33.0 \%\left(\mathrm{Q}_{3}\right)$ of the asfed HCP recipe.

Corn silage was not expected to be difficult to load, as it flows easily during unloading. Corn silage is a relatively inexpensive ingredient and primary component of HCR, representing $26.5\left(\mathrm{Q}_{1}\right)$ to $38.9 \%\left(\mathrm{Q}_{3}\right)$ of the asfed ration. Feeders may not be as careful when loading corn silage compared with more expensive ingredients. Also, the distance between the mixer and the corn silage structure may play a role in the feeder accuracy. Often the corn silage structure is placed far from the mixer, and the feeder will have to make an extra trip to acquire more silage or return leftovers to the structure. It may be easier for the feeder to dispose of the extra feed in the mixer and move to the next ingredient or manually advance if the target was not reached.

Five dairies $(2,5,10,19$, and 25) loaded liquids with an extreme deviation from target weight $\left(Q_{3}:|>75| \mathrm{kg}\right)$. On those dairies, liquids had a deviation from target that ranged from 6.2 to $25.1 \%$. Liquids are added last to the recipe. Often times, the feeder has to get out of the loading equipment and manually open the faucet. The time to load the formulated liquid depends on the pipe design and viscosity of the liquid, especially for 


\begin{tabular}{|c|c|c|c|c|c|c|c|c|c|c|c|c|c|c|c|c|c|c|c|c|c|c|c|c|}
\hline$\nabla$ & fे & \& & $\stackrel{\infty}{\beth}$ & $\stackrel{n}{=}$ & $\vec{\Xi}$ & "े & $\bar{m}$ & $\stackrel{\sim}{\stackrel{\sim}{~}}$ & สี & $\stackrel{\infty}{\sim}$ & ते & 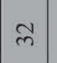 & $\vec{m}$ & 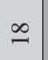 & ते & लै & $F$ & $\stackrel{\infty}{=}$ & $\underline{n}$ & $\stackrel{\sim}{\sim}$ & సิ & in & $\underset{8}{-1}$ & $\underset{\sim}{\stackrel{P}{N}}$ \\
\hline$=$ & ร & নి & 8 & 3 & ત & $m$ & $\stackrel{\sim}{\sim}$ & $n$ & $a$ & $\infty$ & 2 & $\simeq$ & $\infty$ & $\stackrel{\infty}{-1}$ & $r$ & $\cong$ & $\vec{n}$ & m & $\approx$ & $\stackrel{\infty}{\sim}$ & $\vec{m}$ & สั & $m$ & fo \\
\hline s & पे & 8 & in & $\vec{\forall}$ & $\vec{m}$ & $\stackrel{\sim}{\circ}$ & $\dot{m}$ & $\stackrel{\text { त }}{ }$ & $\approx$ & J & $\exists$ & ๗ี & $\underset{\sim}{\infty}$ & $\overrightarrow{0}$ & $\approx$ & $\stackrel{\infty}{\sim}$ & f & $\Xi$ & ๙ & $m$ & iे & $\hat{\infty}$ & 8 & ช \\
\hline$\Xi$ & 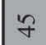 & ले & 5 & ల & $\stackrel{\sim}{\sim}$ & $\cong$ & $\propto$ & $\mathscr{N}_{\sim}$ & $\simeq$ & $=$ & $\underline{n}$ & $a$ & $\infty$ & $a$ & $\tilde{N}$ & m & in & $\vec{n}$ & シ & F & $m$ & $\underset{\sim}{ \pm}$ & ટે & $\stackrel{\circ}{m}$ \\
\hline 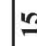 & $\bar{F}$ & $\bar{m}$ & $F$ & \& & $\stackrel{\infty}{\sim}$ & \pm & $\bar{m}$ & n & $\circ$ & 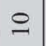 & $\simeq$ & 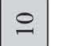 & $a$ & 0 & $a$ & $\infty$ & กิ & f & $\tilde{n}$ & $\vec{n}$ & $\stackrel{\infty}{m}$ & $\vec{\sim}$ & \& & in \\
\hline$r$ & F & 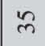 & $\stackrel{\infty}{+}$ & సે & $\approx$ & $a$ & 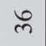 & $\hat{\sim}$ & $\stackrel{-}{\circ}$ & $\simeq$ & $\simeq$ & $\infty$ & $a$ & $\cong$ & $=$ & $\infty$ & $\approx$ & $\stackrel{\infty}{+}$ & 8 & $\underset{m}{\infty}$ & $\bar{m}$ & ส & 于 & m \\
\hline 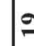 & d & $\stackrel{\infty}{\sim}$ & 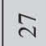 & $\approx$ & ¿ & $\mathscr{F}$ & $m$ & $\stackrel{\infty}{\infty}$ & $a$ & $\underline{2}$ & $a$ & $r$ & $a$ & $\mathcal{F}$ & $\simeq$ & 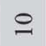 & $m$ & $\bar{F}$ & లn & $\hat{\imath}$ & $\bar{m}$ & $\ddot{\infty}$ & in & ले \\
\hline 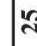 & $\tilde{\imath}$ & $\approx$ & $\ddot{\lambda}$ & $\vec{\lambda}$ & $\stackrel{\infty}{\sim}$ & $\stackrel{\sim}{\sim}$ & స & $\bar{N}$ & $=$ & \pm & $a$ & $\simeq$ & $\simeq$ & $\stackrel{\infty}{\infty}$ & $\approx$ & $\simeq$ & " & f & $m$ & $m$ & $m$ & $\tilde{\infty}$ & से & ले \\
\hline$\approx$ & ల & ल & $\dddot{f}$ & $\tilde{v}$ & $\vec{m}$ & $\varrho$ & 1 & I & $n$ & 인 & 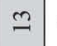 & $r$ & $a$ & - & 1 & 1 & $\tilde{f}$ & f & in & 이 & $F$ & $\stackrel{\infty}{=}$ & I & 1 \\
\hline- & خे & $\hat{\sim}$ & ते & तి & $\stackrel{\bullet}{\ddots}$ & $\bar{m}$ & \pm & J & $=$ & $=$ & $\simeq$ & $a$ & $\infty$ & \pm & $\infty$ & $=$ & $F$ & ले & $\mathcal{F}$ & ஜ & $\approx$ & $\stackrel{+}{+}$ & ป & in \\
\hline o & ন & लै & $\bar{m}$ & $\because$ & 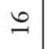 & $\sigma$ & in & తु & $r$ & $\underline{\simeq}$ & 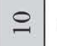 & $r$ & 0 & $\nabla$ & \pm & $=$ & $\vec{m}$ & f & $\mathscr{f}$ & ন & $\widetilde{\Xi}$ & $a$ & $\widehat{6}$ & $\infty$ \\
\hline$\approx$ & $\Xi$ & $F$ & లి & $\simeq$ & $\approx$ & ন & $=$ & $\leadsto$ & $n$ & 0 & $=$ & 인 & $a$ & $\simeq$ & 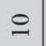 & $\cong$ & $\mathcal{A}$ & $\bar{f}$ & $\mathcal{F}$ & $\widetilde{N}$ & $\widetilde{N}$ & $\stackrel{ల}{n}$ & $\approx$ & ‡ \\
\hline ת & 2 & $\bar{\sim}$ & $\tilde{\epsilon}$ & \pm & 1 & $\simeq$ & 寸 & $=$ & $\stackrel{\circ}{-}$ & $\cong$ & 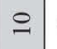 & $a$ & 1 & $\mathrm{I}$ & 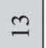 & $\infty$ & ते & $\dot{m}$ & $m$ & $\ddot{\lambda}$ & I & 1 & in & i \\
\hline$=$ & $\because$ & กิ & กิ & $\stackrel{\infty}{\sim}$ & 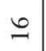 & 1 & $\bar{\sim}$ & $=$ & $a$ & $a$ & $\varrho$ & $\infty$ & $a$ & $a$ & $a$ & $a$ & $\stackrel{ \pm}{ \pm}$ & ஓ & ిల & $\stackrel{i}{2}$ & i & $\widetilde{N}$ & $\vec{m}$ & $\bar{\imath}$ \\
\hline 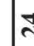 & $\stackrel{\infty}{\sim}$ & \pm & లి & \pm & & $\infty$ & $\approx$ & $\cong$ & 0 & $r$ & $a$ & 6 & in & in & $r$ & 6 & సे & $\tilde{N}$ & f & ¿े & $\stackrel{0}{\circ}$ & $\stackrel{m}{-}$ & $m$ & $\vec{\sim}$ \\
\hline 。 & $\cong$ & $\approx$ & $\bar{\imath}$ & \pm & $\Rightarrow$ & $\hat{n}$ & 1 & 1 & $\stackrel{-}{\circ}$ & \pm & $=$ & $\infty$ & 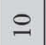 & 2 & 1 & 1 & $\tilde{N}$ & m & लै & ส & $\widetilde{N}$ & 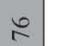 & 1 & 1 \\
\hline 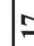 & 0 & సे & సి & $a$ & $\simeq$ & $\underline{n}$ & \pm & $\mathscr{0}$ & 6 & $a$ & $\infty$ & 6 & 0 & $\theta$ & in & 0 & $\ddot{\sim}$ & ले & ঙ & $\stackrel{0}{0}$ & $\underset{\sim}{J}$ & $\stackrel{\infty}{-1}$ & ণ & $\ddot{\sim}$ \\
\hline$=$ & \pm & $\ddot{\imath}$ & 2 & $=$ & + & - & $r$ & F & + & in & $r$ & + & + & - & + & $r$ & $\infty$ & নे & $\hat{\sim}$ & $\cong$ & $a$ & $m$ & $\simeq$ & फ़ \\
\hline$=$ & \pm & $\propto$ & กิ & 1 & 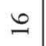 & $r$ & 1 & I & $\simeq$ & $\simeq$ & \pm & 1 & $\underline{m}$ & $\infty$ & 1 & 1 & $\hat{\sim}$ & $\ddot{m}$ & $m$ & I & లి & f & I & 1 \\
\hline 6 & \pm & $=$ & \pm & \pm & $\simeq$ & ते & \pm & I & $\infty$ & $=$ & $\infty$ & $\infty$ & 0 & $a$ & $\infty$ & 1 & $\stackrel{N}{*}$ & $\tilde{\sim}$ & $\approx$ & $\approx$ & $\simeq$ & $\stackrel{\infty}{m}$ & $\approx$ & 1 \\
\hline$\propto$ & $\simeq$ & t & $\therefore$ & in & $n$ & 1 & $n$ & 0 & $\sigma$ & \pm & $a$ & $m$ & $m$ & 1 & 0 & $N$ & 0 & ले & নे & $a$ & $a$ & 1 & $=$ & $a$ \\
\hline$\propto$ & $\cong$ & $\cong$ & $\cong$ & $n$ & $\cong$ & $a$ & \pm & 1 & 0 & $a$ & - & t & 0 & $\cong$ & 0 & 1 & $\approx$ & $\approx$ & ส & $\cong$ & 2 & ส & $\vec{\sim}$ & 1 \\
\hline 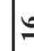 & $\cong$ & $\because$ & $\cong$ & $m$ & $\infty$ & $a$ & $r$ & I & $m$ & 0 & 0 & $\theta$ & $\nabla$ & $m$ & $\nabla$ & 1 & $\cong$ & $\approx$ & $\widetilde{N}$ & $\stackrel{\infty}{\sim}$ & $m$ & $m$ & $=$ & 1 \\
\hline$\hat{\jmath}$ & + & \pm & \pm & + & + & $\simeq$ & \pm & $a$ & + & $\infty$ & $n$ & $\theta$ & $\nabla$ & in & $\infty$ & 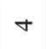 & $a$ & $\tilde{A}$ & $\vec{\sim}$ & $a$ & $a$ & $\ddot{\lambda}$ & $\approx$ & $\cong$ \\
\hline 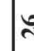 & $=$ & 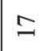 & \pm & 6 & $=$ & 0 & $\infty$ & $\sigma$ & 0 & $a$ & 0 & $n$ & $n$ & $\sigma$ & $\nabla$ & in & 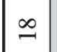 & i & సి & $\cong$ & $=$ & $=$ & 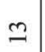 & $\stackrel{ }{\circ}$ \\
\hline ल & $\mathrm{N}$ & 6 & 0 & $m$ & 0 & $\mathrm{~N}$ & 0 & - & + & $n$ & $n$ & $m$ & $m$ & - & + & $m$ & 6 & $\cong$ & 6 & $r$ & + & + & + & in \\
\hline 菅 & 希 & 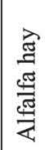 & 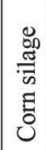 & 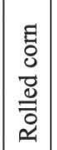 & 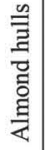 & 量 & 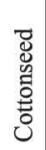 & 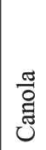 & 忞 & 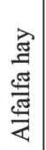 & 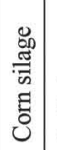 & 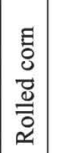 & 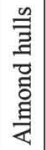 & 量 & 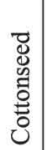 & $\begin{array}{l}\text { 평 } \\
\text { छ్ }\end{array}$ & 总 & 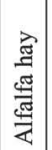 & 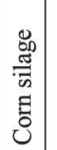 & 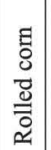 & 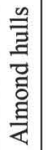 & 穿 & 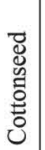 & $\begin{array}{l}\text { శే } \\
\text { స్ }\end{array}$ \\
\hline & \multicolumn{8}{|c|}{ 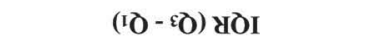 } & \multicolumn{8}{|c|}{ 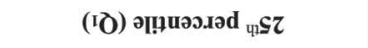 } & \multicolumn{8}{|c|}{ 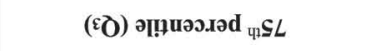 } \\
\hline
\end{tabular}

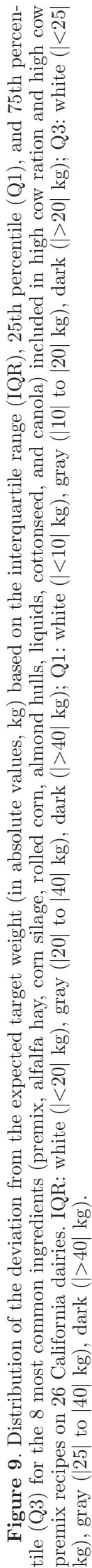


molasses in winter. Our results indicate that on these dairies the feeder might often forget to close the faucet in time.

\section{CONCLUSIONS}

Opportunities to improve feeder performance were observed based on loading errors. The TL settings introduced an important deviation from target weight for some ingredients. Dairy producers should evaluate if readjusting the TL settings for some ingredients could reduce the deviation from target. Deviation from target may be influenced by ingredient type. Some ingredients (rolled corn and almond hulls) were loaded with mostly adequate accuracy and precision, whereas others (alfalfa hay, corn silage, and canola) were mostly loaded with poor accuracy and precision. Our results indicated that some dairies were able to load ingredients with minimal deviation from target weight, suggesting that some poor-performing dairies could set higher goals for loading accuracy and precision on their operations.

\section{ACKNOWLEDGMENTS}

Financial support for this research was partially provided by the Center for Food Animal Health at University of California-Davis School of Veterinary Medicine and Barrie de la Maza Scholarship. The authors appreciate the collaboration of participating dairies for allowing us to use their records. A special appreciation is extended to Manuel Soares, Craig Lew, Jose García, and Cristian Medina (Valley Agriculture Software) for training us on the use of the FMS. Also, we are very appreciative for the insight that dairy nutritionist and consultants offered to us, specifically Phil Jardon, Chel Moore, and Miguel Morales (Elanco Animal Health), Matt Budine and Enrique Schcolnik (Progressive Dairy Solutions), Alejandro Castillo (University of California Cooperative Extension), Aaron Highstreet (Highstreet Dairy Consulting), Tyler Colburn (Alpha Dairy Consulting), Doug DeGroff (Diversified Dairy Solutions), and Chris Dei (Sierra Vista Dairy Consulting).

\section{REFERENCES}

Buckmaster, D. R., and L. D. Muller. 1994. Uncertainty in nutritive measurements of mixed livestock rations. J. Dairy Sci. 77:37163724

CDFA. 2014. California Department of Food and Agriculture-California cost of milk production 2014 Annual. Accessed Jan. 30, 2016. http://www.cdfa.ca.gov/dairy/dairycop_annual.html.

James, R. E., and B. Cox. 2008. Feeding management to reduce the environmental impact of dairy farms. Pages 31-42 in Proc. 45th Florida Dairy Prod. Conf., University of Florida, Gainesville. University of Florida Press, Gainesville.

Rossow, H. A., and S. S. Aly. 2013. Variation in nutrients formulated and nutrients supplied on 5 California dairies. J. Dairy Sci. 96:7371-7381.

Silva-del-Río, N., and A. R. Castillo. 2012. Degree of agreement between the ration formulated and the ration fed on seven California dairies. J. Dairy Sci. 95(E. Suppl. 2):579. (Abstr.)

Silva-del-Río, N., J. M. Heguy, and A. Lago. 2010. Feed management practices on California dairies. J. Dairy Sci. 93(E-Suppl.):773. (Abstr.)

Sova, A. D., S. J. LeBlanc, B. W. McBride, and T. J. DeVries. 2014 Accuracy and precision of total mixed rations fed on commercial dairy farms. J. Dairy Sci. 97:562-571.

St-Pierre, N. R., and W. P. Weiss. 2015. Partitioning variation in nutrient composition data of common feeds and mixed diets on commercial dairy farms. J. Dairy Sci. 98:5004-5015.

Stewart, B. A., R. E. James, K. F. Knowlton, M. L. McGilliard, and M. D. Hanigan. 2011. Case Study: An example of application process control charts to feed management on dairy farms. Prof. Anim. Sci. 27:571-573. 